\title{
Nanoscale Periodic Morphologies on the Fracture Surface of Brittle Metallic Glasses
}

\author{
G. Wang, ${ }^{1}$ D. Q. Zhao, ${ }^{1}$ H. Y. Bai, ${ }^{1}$ M. X. Pan, ${ }^{1}$ A. L. Xia, ${ }^{1}$ B. S. Han,${ }^{1}$ X. K. Xi,${ }^{2}$ Y. Wu, ${ }^{2}$ and W. H. Wang ${ }^{1} *$ \\ ${ }^{1}$ Institute of Physics, Chinese Academy of Sciences, Beijing 100080, People's Republic of China \\ ${ }^{2}$ Department of Physics and Astronomy, University of North Carolina, Chapel Hill, North Carolina 27599-3255, USA
}

(Received 20 September 2006; published 5 June 2007)

\begin{abstract}
Out-of-plane, nanoscale periodic corrugations are observed in the dynamic fracture surface of brittle bulk metallic glasses with fracture toughness approaching that of silica glasses. A model based on the meniscus instability and plastic zone theory is used to explain such dynamic crack instability. The results indicate that the local softening mechanism in the fracture is an essential ingredient for controlling the formation of the unique corrugations, and might provide a new insight into the origin of fracture surface roughening in brittle materials.
\end{abstract}

The fracture surface roughening phenomena of brittle glasses have generated much recent interest [1-4]. The interaction between the crack front and acoustic waves emitted from the dynamic crack propagation is regarded as the main cause for leaving roughness and undulations [1-6]. However, the detailed origin of the corrugations on the fracture surface and the related energy dissipation mechanism are still unclear. Recently, brittle bulk metallic glasses (BMGs) [7], which have extremely low value of notch fracture toughness $\left(K_{C}\right)\left(<2 \mathrm{MPa} \mathrm{m}^{1 / 2}[8,9]\right)$, have been developed. The atomic-scale disorder in BMGs has significant structural difference from those of nonmetallic glasses. The brittle BMGs then can act as a new model to study the features of crack propagation $[9,10]$. In this work, we investigate the fracture behavior of typical brittle $\mathrm{Mg}_{65} \mathrm{Cu}_{25} \mathrm{Gd}_{10}$ and $\mathrm{Dy}_{40} \mathrm{Y}_{16} \mathrm{Al}_{24} \mathrm{Co}_{20}$ BMGs with different mechanical properties, such as yielding strengths $\left(\sigma_{Y}\right)$, elastic moduli $(E)$, and Poisson's ratios $(\nu)$, but similar $K_{C}$ values of 1-2 MPa m ${ }^{1 / 2}$ [8]. The fractographies of the specimens show a flat mirror surface at microscale, while remarkable periodic corrugation patterns at nanoscale are observed by scanning electron microscopy (SEM) and atomic force microscopy (AFM). The mechanism for the formation of the periodic corrugation is quantitatively discussed.

The fracture surfaces of cylindrical BMGs $(\phi 3 \times 20 \mathrm{~mm})$ with single-edge notch were prepared by three-point bending tests on an Instron 4505 machine under a constant displacement velocity of $1 \mathrm{~mm} / \mathrm{min}$ under ambient conditions. The $\sigma_{Y}$ and elastic modulus were measured from a compressive test [8]. The newly created fracture surfaces were examined using a Philips XL30 SEM and a Digital NanoScope IIIa D-3000 AFM. The measurements of the shear and longitudinal wave speeds, density, glass transition $\left(T_{g}\right)$, and melting temperatures $\left(T_{m}\right)$ can be referred as in Ref. [7].

The fractographies of the BMGs by SEM from microscale to nanoscale are shown in Fig. 1. In microscale, the fracture surfaces of these BMGs consist of a mirror zone and a river pattern zone [Figs. 1(a) and 1(b)]. However, in nanoscale, the dimple structure appears in the region near the notch [Figs. 1(b) and 1(e)]. The average dimple sizes for the Dy- and Mg-based BMGs are about 77 and $103 \mathrm{~nm}$, respectively. With crack propagating, the dimple structure transforms into a periodic corrugation pattern [Figs. 1(c) and 1(f)]. There are irregular nanoscale wavy corrugations with average spacing of $31 \mathrm{~nm}$ on the mirror zone of Dybased BMG [Fig. 1(c)]. For Mg-based BMG, strikingly, the periodic and straight corrugations (perpendicular to the crack propagation direction) with average spacing of $60 \mathrm{~nm}$ appear on the mirror zone [Fig. 1(f)].

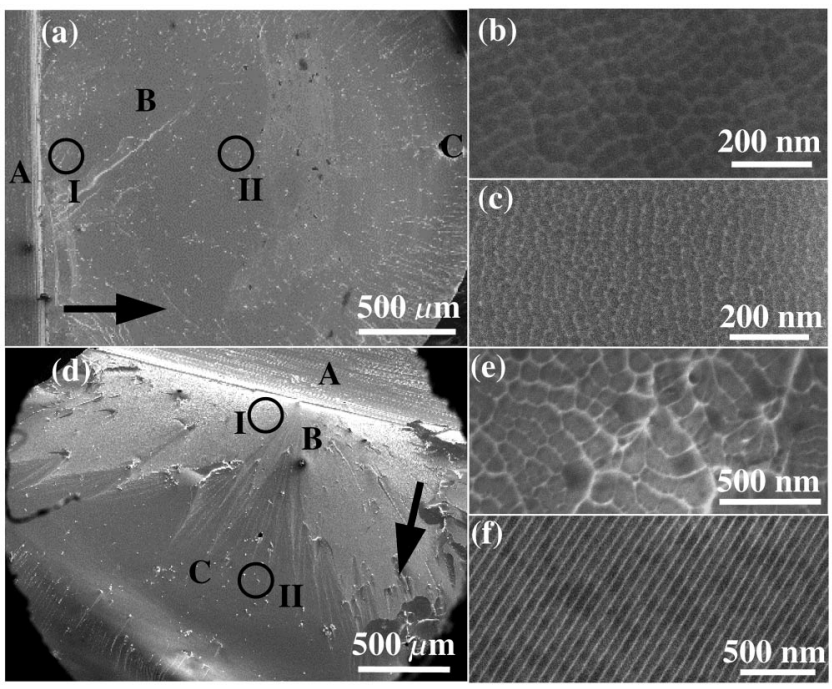

FIG. 1. SEM observation results for fractographies of two BMGs under three-point bending fracture (A, B, and C represent notch position, mirror zone, and river pattern zone). (a) Fractography of the Dy-based BMG. (b) Detail morphology near the notch corresponding to point I in (a). (c) Detail morphology of the mirror zone corresponding to point II in (a). (d) Fractography of the Mg-based BMG. (e) Detail morphology near the notch corresponding to point $I$ in (d). (f) Detail morphology of the mirror zone corresponding to point II in (d). [Arrows in (a) and (d) indicate the crack propagation direction]. 
The AFM observations on the mirror zones are given in Figs. 2(a) and 2(d), in which the fracture surface is on the $X-Z$ plane. The 3D pattern suggests that the fracture surface corrugates not only on the $X-Y$ plane (along the crack propagation direction) but also on the $Y-Z$ plane (perpendicular to the crack propagation direction). The sectional shapes of the corrugation on the $X-Y$ plane are shown in Figs. 2(b) and 2(e), which show a near sinusoidal curve. The corrugation pattern on the Mg-based BMG fracture surface is more regular [Fig. 2(e)] compared with that of the Dy-based BMG [Fig. 2(b)]. For the Dy- and Mg-based BMGs, the average heights (in $Y$ axis) of the corrugations are 3.6 and $6.5 \mathrm{~nm}$, respectively; the average wavelengths on the $X-Y$ plane are 32 and $64 \mathrm{~nm}$, respectively, which coincide with those measured from SEM. Further analysis shows that the corrugation for Dy- and Mg-based BMGs on the $Y$ - $Z$ plane also shows a wavy shape [Figs. 2(c) and 2(f)] with average wavelengths of 65 and $92 \mathrm{~nm}$, respectively. Based on the 3D pattern, the waveform of the corrugation presenting on the $X-Z$ plane is considered to be the same as that on the $Y-Z$ plane.

The matching areas on both fractured surfaces observed both by AFM and SEM are shown in Fig. 3. The observations clearly show a peak-to-peak separation at the crack
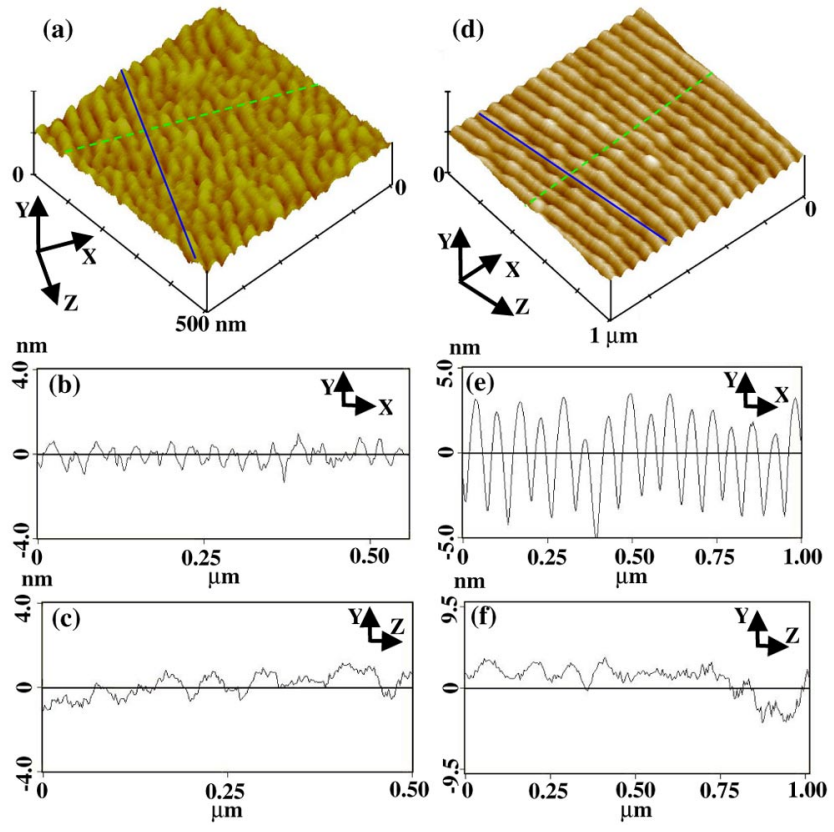

FIG. 2 (color online). AFM results for the mirror zone on the fracture surface of two BMGs. Cracks propagate along the $X$ direction. The corrugation height was measured by Nanoscope III software.) (a) 3D image for the Dy-based BMG. (b) Section shape of the corrugation being along a dashed line in (a), i.e., the crack propagation direction. (c) Section shape of the corrugation being along a solid line in (a), i.e., perpendicular to the crack propagation direction. (d) 3D image for the Mg-based BMG. (e) Section shape of the corrugation being along a dashed line in (d), i.e., the crack propagation direction. (f) Section shape of the corrugation being along a solid line in (d), i.e., perpendicular to the crack propagation direction. tip (for comparison, we chose the area with some flaws as the reference point). The results indicate that local plastic deformation plays a dominant role in blunting initially sharp cracks where progressive local separation still occurs by a sequence of vein pattern formation, followed by plastic growth and linking of such vein patterns or coalescence of damage cavities along the extension of the crack plane. A sketch of the crack tip plastic separation process is drawn in Fig. 4(a).

$\mathrm{X}$-ray diffraction analysis of the fracture surface shows the glassy nature, which excludes the influence of crystallization on the fractographies. Crack front wave is a factor bringing out the roughness on the fracture surface of brittle materials [11]. However, the observed phenomenon in the BMG is not the crack front waves because the periodic perturbations cannot occur in BMGs with homogenous microstructure and without periodic structural asperities. The oscillatory wavelength for Wallner lines [12] is $W=$ $\left(2 h V / c_{l}\right) / \sqrt{1-\left(V / c_{l}\right)^{2}}$, where $h$ is the distance from the crack tip to the boundary, $V$ is the crack propagating speed, and $c_{l}$ is the longitudinal wave speed [13]. For the BMGs, the $V$ is estimated to be $(1.3-5.5) \times 10^{-3} \mathrm{~m} / \mathrm{s}$, which are unreasonably low for generating Wallner lines [14]. Thus, the observed periodic corrugations in BMGs cannot be attributed to the Wallner lines.
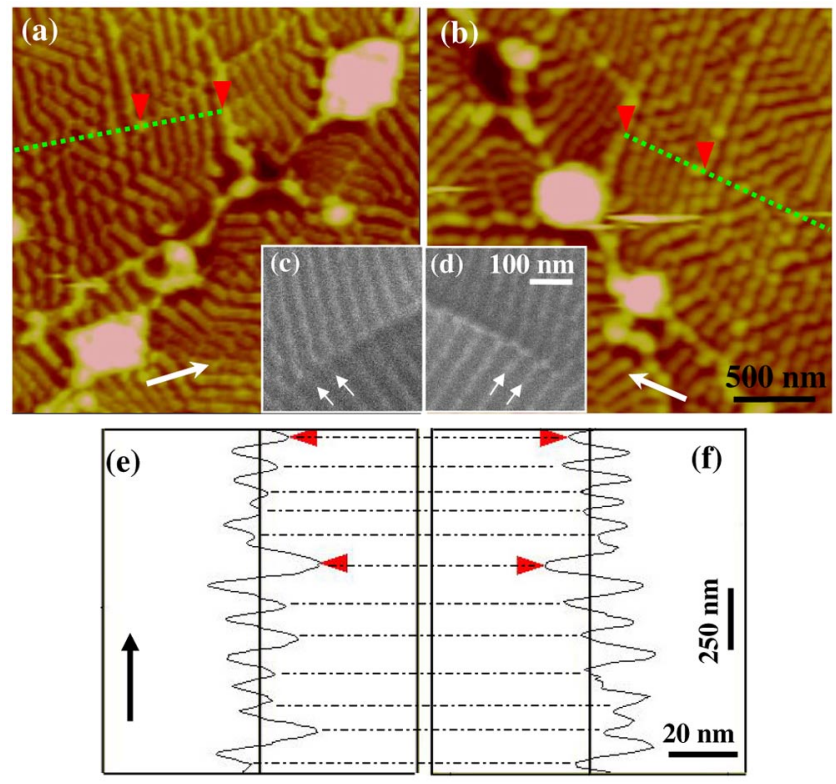

FIG. 3 (color online). (a),(b) A comparison of left and right surfaces of the Mg-based BMG observed by AFM. (Arrows indicate the crack propagation direction. Triangles point out the reference points that indicate the peak-to-peak match.) (c),(d) The comparison of left and right surfaces of the Mgbased BMG by SEM (arrows are guidelines for peak-to-peak matching). (e),(f) Section shapes of the corrugations being along dotted lines in (a) and (b). (The arrow indicates the crack propagation direction.) Dash-dotted lines clearly indicate the peak-to-peak match. 
TABLE I. The parameters of $T_{g}, T_{m}, \sigma_{Y}$, elastic moduli $(E)$, longitudinal wave speeds $\left(c_{l}\right)$, shear wave speeds $\left(c_{s}\right), \rho$, and Poisson ratios $(\nu)$ of the BMGs.

\begin{tabular}{lcccccccc}
\hline \hline \multicolumn{1}{c}{ BMGs } & $T_{g}(\mathrm{~K})$ & $T_{m}(\mathrm{~K})$ & $\sigma_{Y}(\mathrm{MPa})$ & $E(\mathrm{GPa})$ & $c_{s}(\mathrm{~m} / \mathrm{s})$ & $c_{l}(\mathrm{~m} / \mathrm{s})$ & $\rho\left(\mathrm{g} / \mathrm{m}^{3}\right.$ & $\nu$ \\
\hline $\mathrm{Dy}_{40} \mathrm{Y}_{16} \mathrm{Al}_{24} \mathrm{Co}_{20}$ & 631 & 978 & 1110 & 56.5 & 1839 & 3552 & $7.20 \times 10^{6}$ & 0.317 \\
$\mathrm{Mg}_{65} \mathrm{Cu}_{25} \mathrm{Gd}_{10}$ & 424 & 717 & 672 & 50.6 & 2254 & 4319 & $3.79 \times 10^{6}$ & 0.313 \\
$\mathrm{Fe}_{48} \mathrm{Cr}_{15} \mathrm{Mo}_{14} \mathrm{Er}_{2} \mathrm{C}_{15} \mathrm{~B}_{6}$ & $\cdots$ & 1373 & 3800 & 205.0 & $\cdots$ & $\cdots$ & $\cdots$ & 0.300 \\
\hline \hline
\end{tabular}

Local temperature rise accompanying plastic deformation near the crack tip for BMGs has been confirmed by previous studies [15-17]. The maximum temperature rise $\Delta T$ within the plastic process zone can be estimated as [18] $\Delta T=\left[1.414\left(1-\nu^{2}\right) K_{c} \sigma_{Y} \sqrt{V}\right] /(E \sqrt{\rho C k})$, where $\rho$ is the density, $C$ is the thermal capacity, and $k$ is the thermal conductivity. For BMGs, $C \approx 1 \mathrm{~J} / \mathrm{gK}$ [19] and $k \approx$ $5 \mathrm{~W} / \mathrm{mK}$ [20]. The other parameters are listed in Table I. The maximum crack speed $V<V_{R} \approx 0.9225 c_{s}$, where $c_{s}$ is the shear wave speed [14]. Thus, the $\Delta T$ for the Dy- and Mg-based BMGs is estimated to be 343 and $355 \mathrm{~K}$, respectively, which are higher than their $T_{g}$ while much lower than their $T_{m}$. For comparison, the $\Delta T$ of $\mathrm{Zr}_{41.25} \mathrm{Ti}_{13.75} \mathrm{Ni}_{10} \mathrm{Cu}_{12.5} \mathrm{Be}_{22.5}$ BMG (Vit1) is estimated to be $\sim 10^{4} \mathrm{~K}$, which is much higher than its $T_{m}$ and in accordance with the measurement [17]. The experimental and analysis results indicate that the fracture in brittle BMGs is capable of undergoing inelastic deformation which results in a variant of the meniscus instability happening in the crack tip [21].

The relationship of $K_{\mathrm{IC}}$ and the crack tip open distance (CTOD) was established as [22] $K_{\mathrm{IC}}=$ $\sqrt{(\mathrm{CTOD})} \pi \sigma_{Y} E / 2.7$. Based on the sketch of the crack tip separation process [Fig. 4(a)], the CTOD for the BMGs can be roughly estimated as the double height of their corrugations. The calculated $K_{\mathrm{IC}}$ values of the two BMGs, which are about $0.72 \mathrm{MPa} \mathrm{m}^{1 / 2}$, agree well with the measurement $\left(1-2 \mathrm{MPa} \mathrm{m}^{1 / 2}\right)$ [8]. The agreement indicates that the meniscus instability of the crack tip controls the crack propagation.

An infinitesimal perturbation with an initial wavelength $\lambda$ on the meniscus advancing into the crack front is shown in Fig. 4(a). Once $\lambda$ is larger than a critical value, i.e., $(d \sigma / d x) \geq \chi(2 \pi / \lambda)^{2}$, where $\chi$ is the surface tension (for BMGs, $\chi \approx 1 \mathrm{~J} / \mathrm{m}^{2}$ [9]), and $d \sigma / d x$ is the negative pressure gradient in the front of the crack tip $[d \sigma / d x=$ $\left(2 \pi \sigma_{\text {th }}\right) / \delta$, where $\sigma_{\text {th }}$ is the theoretical strength $\left(\sigma_{\text {th }} \cong\right.$ $E / 10)]$, and $\delta$ is a range parameter, $\delta \approx 0.1-0.4 \mathrm{~nm}$ for typical brittle solids $[10,23]$, the perturbation will further grow to form a final corrugation on the $X-Z$ plane $[21,22]$. For Dy- and Mg-based BMGs, $d \sigma / d x$ are estimated to be $89 \times 10^{18}$ and $79 \times 10^{18} \mathrm{~Pa} / \mathrm{m}$, and $\chi(2 \pi / \lambda)^{2}$ are $0.1 \times$ $10^{18} \mathrm{~Pa} / \mathrm{m}$ and $0.07 \times 10^{18} \mathrm{~Pa} / \mathrm{m}$, respectively. It is obvious that the instability criteria in these BMGs are satisfied. For the Dy- and Mg-based BMGs, the wavelengths of their final corrugation on the $X-Z$ plane are $\lambda_{\text {Dy }}=65$ and $\lambda_{\mathrm{Mg}}=92 \mathrm{~nm}$, which can be roughly considered as the wavelength of the initial perturbation of the meniscus. The small-scale yielding model indicates that the fracture surface separation process is confined to a frontal plastic zone [10]. The radius of the plastic zone $(R)$ for plane strain is [24]: $R=1 / 6 \pi\left(K_{I} / \sigma_{Y}\right)^{2}$. The $R$ values for the Dy- and Mg-based BMGs are calculated to be 22.3 and $60.9 \mathrm{~nm}$, respectively. The $R$ values are much smaller than their corresponding $\lambda$, indicating that the maximum stress presenting in the plastic zone does not exceed the wavelength of the corrugation and then the initial perturbation actually does not develop.

If $R$ is larger than $\lambda$, this instability should induce the dimple structure. The dimple structure near the notch can be treated as the wavelength of the final corrugation. The CTODs near the notch estimated from AFM measurements are 21.6 and $22.4 \mathrm{~nm}$ for Dy- and Mg-based BMGs, respectively, and then the $R$ values are calculated to be $R_{\mathrm{Dy}}=89$ and $R_{\mathrm{Mg}}=104 \mathrm{~nm}$, which are larger than or at least equal to the dimple size. The wavelength of the final corrugation should be close to that of the initial perturbation [22]. As a result, once $R \geq \lambda$, the meniscus instability will develop into the dimple structure confined in the plast-

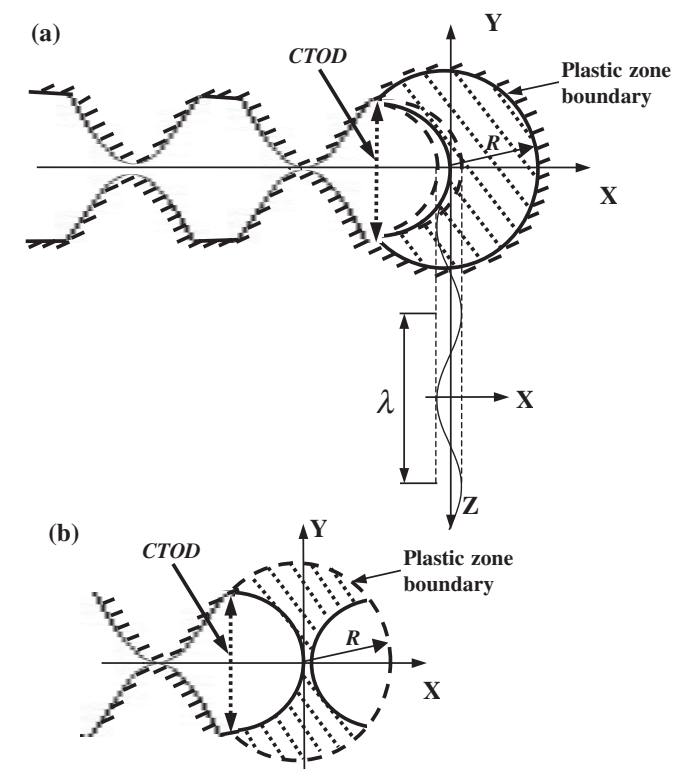

FIG. 4. (a) Sketch illustrating the crack tip opening mechanism (crack propagates along the $X$ direction). Crack extension by the peak-to-peak separation of the corrugation on the $X-Y$ plane and the infinitesimal perturbation on the crack front on the $X-Z$ plane ( $\lambda$ is the wavelength of the initial perturbation). (b) The microvoid formation mechanism in the plastic zone. 
ic zone. As the maximum stress on the front of the crack tip reaches the yield stress of the materials, the microvoid will be stimulated as shown in Fig. 4(b) [24]. When the microvoid reaches the size of the plastic zone, the neighboring microvoids assemble together and then the corrugation forms. Thus, the spacing of the corrugations or the size of the dimple structure is close to $R$. This is also the reason that tough BMGs such as Vit1 with a larger plastic zone of $\sim 120 \mu \mathrm{m}$ in size show a radial corrugation pattern rather than the periodic corrugation tested under similar conditions (which is the direct evidence of the necking and meniscus instability [9]). The $R$ value of Vit1 is about 100 times larger than its size of final corrugation $(\sim 1.3 \mu \mathrm{m})$ [25]. This is also supported by the fact that the fracture surfaces of the BMG in supercooled state do not exhibit the periodic corrugations because the elevated temperature influences the size of the plastic zone.

Near the notch, the crack speed is not set up, and the fracture can be considered a quasistatic behavior. With the crack propagating, the dynamic process results in the changes of fractography from the dimple to the periodic corrugation. The increase of the crack velocity is accompanied by the reduction of the values of local $K_{\mathrm{IC}}$ and $R$, and the spacing of the periodic corrugation is reduced from about 80 to $35 \mathrm{~nm}$. When $R<\lambda$, the plastic zone restrains the development of the meniscus instability. This restraint is more significant in brittle BMGs with lower $K_{C}$ or smaller plastic zone, because the smaller plastic zone is more easily disturbed by the dynamic process. Therefore, the periodic corrugation presents on the fracture surface of brittle BMGs.

We have investigated the fractographies of a $\mathrm{Fe}_{48} \mathrm{Cr}_{15} \mathrm{Mo}_{14} \mathrm{Er}_{2} \mathrm{C}_{15} \mathrm{~B}_{6}$ BMG with similar $K_{C}$ but markedly different $T_{m}, \sigma_{Y}, E$ (see Table I). The mirror zone of the BMG exhibits a featureless pattern within the SEM resolution limit of $1.5 \mathrm{~nm}$. This is due to its fracture process possibly behaves the pure brittle fracture, in which, at the tip is of a very sharp crack, the cohesive strength of the solid is reached before any plastic deformation can be stimulated. The plastic zone of the Fe-based BMG is less than $8 \mathrm{~nm}$ in radius. Most of the solids behave as a completely brittle fracture when the ratios of $T_{t} / T_{m} \leq 0.3$ $[19,26]$, where $T_{t}$ is the test temperature $\left(T_{t}=300 \mathrm{~K}\right)$. The $T_{t} / T_{m}$ for the Fe-, Dy-, and Mg-based BMGs are $0.22,0.31$, and 0.42 , respectively. The $T_{t} / T_{m}$ for the Febased BMG without the periodic corrugation pattern is less than 0.3 in which any thermally activated process is absent [26]. While for the Dy- and Mg-based BMGs, the $T_{t} / T_{m}$ is larger than 0.3 and the plastic zone can be formed by transforming the work of the fracture to the crack tip front. The results further confirm that the local plastic deformation and the size of the fracture zone at the crack tip play a significant role in the formation of the nanoscale corrugation.

In summary, we observed the nanoscale periodic corrugation on the fracture surface of brittle BMGs in which the fracture still proceeds through the local softening mecha- nism. We demonstrate that the periodic nanoscale roughing is a result of a local plastic fracture in which local plasticity plays a dominant role to blunt the crack tip but where progressive local separation still occurs. The size of the plastic zone affected by the crack speed and toughness of the glasses is a key parameter for dominating the fractographies. This indicates that it is an essential ingredient in the dynamic fracture of brittle BMGs to have a critical range for forming the periodic corrugation.

Financial support from the NSFC (No. 50621061) is acknowledged.

*Corresponding author.

Electronic address: whw@aphy.iphy.ac.cn

[1] J.-P. Guin and S. M. Wiederhorn, Phys. Rev. Lett. 92, 215502 (2004); J. Feinberg, S. Gross, M. Marder, and H. Swinney, Phys. Rev. Lett. 67, 457 (1991).

[2] D. Bonamy and K. Ravi-Chandar, Phys. Rev. Lett. 93, 099602 (2004).

[3] J. E. Field, Contemp. Phys. 12, 1 (1971).

[4] S. Ramanathan and D. S. Fisher, Phys. Rev. Lett. 79, 877 (1997).

[5] E. Bouchaudrevert et al., J. Mech. Phys. Solids 50, 1703 (2002).

[6] B. N. Cox, H. Gao, D Gross, and D. Rittel, J. Mech. Phys. Solids 53, 565 (2005).

[7] X. K. Xi et al., Intermetallics 13, 638 (2005); W. H. Wang et al., Mater. Sci. Eng., R 44, 45 (2004).

[8] W. H. Wang, J. Appl. Phys. 99, 093506 (2006).

[9] X. K. Xi et al., Phys. Rev. Lett. 94, 125510 (2005).

[10] B. Lawn, Fracture of Brittle Solids (Cambridge University Press, Cambridge, 1993), 2nd ed., pp. 53-174.

[11] E. Sharon, G. Cohen, and J. Fineberg, Nature (London) 410, 68 (2001); Phys. Rev. Lett. 88, 085503 (2002).

[12] D. Bonamy and K. Ravi-Chandar, Phys. Rev. Lett. 91, 235502 (2003).

[13] R. D. Deegan et al., Phys. Rev. Lett. 88, 014304 (2001).

[14] K. Ravi-Chandar and B. Yang, J. Mech. Phys. Solids 45, 535 (1997).

[15] F. Spaepen, Acta Metall. 25, 407 (1977).

[16] B. Yang et al., Appl. Phys. Lett. 86, 141904 (2005).

[17] J. J. Lewandowski and A.L. Greer, Nat. Mater. 5, 15 (2006).

[18] A. S. Argon, The Physics of Strength and Plasticity (MIT Press, Cambridge, MA, 1969), p. 286.

[19] C. T. Liu et al., Metall. Mater. Trans. A 29, 1811 (1998).

[20] M. Yamasaki, S. Kagao, and Y. Kawamura, Scr. Mater. 53, 63 (2005).

[21] G. I. Taylor, Proc. R. Soc. A 201, 192 (1950).

[22] A. S. Argon and M. Salama, Mater. Sci. Eng. 23, 219 (1976).

[23] T. H. Courtney, Mechanical Behavior of Materials (Overseas Press India Private Limited, New Delhi, 2006), p. 410.

[24] D.P. Miannay, Fracture Mechanics (Springer-Verlag, New York, 1998), p. 53.

[25] G. Wang et al., Intermetallics 13, 642 (2005).

[26] A. Kelly, W. R. Tyson, and A. H. Cottrell, Philos. Mag. 15, 567 (1967). 